Artículo científico / Scientific article

Los textos publicados son responsabilidad exclusiva de sus autores

\title{
Efectividad del Módulo Introductorio de Formación de la Superintendencia de Administración Tributaria
}

\section{Effectiveness of the Introductory Training Module of the Superintendencia de Administración Tributaria}

\author{
- Lidia Velásquez, Miriam Hernández y Patricia Mazariegos \\ - Facultad de Humanidades \\ Universidad de San Carlos de Guatemala \\ lidia.2312@hotmail.com \\ https://orcid.org/0000-0002-6210-3430
}

\section{Referencia del artículo}

Velásquez, L., Hernández, M. y Mazariegos, P. (2020). Efectividad del Módulo Introductorio de Formación de la Superintendencia de Administración Tributaria. Revista Guatemalteca de Educación Superior, 3(1), 19-31. DOI: https://doi.org/10.46954/revistages.v111.2

Recibido 28/03/2020.

Revisado 29/03/2020.

Aceptado 26/06/2020.

\section{Resumen}

En esta investigación se determinó la efectividad del Módulo Introductorio de Formación (MIF) en las categorías ocupacionales de la Superintendencia de Administración Tributaria (SAT). Se utilizó un diseño cuantitativo de tipo no experimental transeccional. Se aplicó una encuesta y se hizo el cálculo de la $T$ de Student de una base de datos generada por el investigador a partir de los registros de datos personales, rendimiento académico y categorías ocupacionales para determinar la efectividad del MIF de acuerdo con el modelo de evaluación de Donald Kirkpatrick y los criterios de calidad de la ISO 9126. Como resultados se evidencia que hay efectividad en la realización del MIF, se evidencia que hubo aprendizaje al no haber diferencia estadísticamente significativa entre las medias 
Facultad de Humanidades

Escuela de Estudios de Postgrado

Palabras clave: efectividad, evaluación, estándar ISO

Keywords: effectiveness, evaluation, ISO Standard obtenidas por los sujetos de estudio antes y después de haber realizado el MIF. Existen cambios en la conducta en la aplicación de los conocimientos, habilidades y actitudes como la práctica de los valores institucionales y una cultura de servicio, así como la experiencia en la realización del curso en un entorno virtual en donde el software cumple con los requerimientos de la ISO 9126. Por tanto, utilizar este modelo, al igual que los criterios que establece la ISO 9126, por lo que, utilizar tanto el modelo de Kirkpatrick como la ISO 9126 resultan herramientas útiles para las organizaciones que puedan determinar la efectividad de la formación que reciben sus colaboradores.

\section{Abstract}

This research determined the effectiveness of the Introductory Training Module (MIF) in the occupational categories of the Superintendence of Tax Administration (SAT). A quantitative design of a non-experimental transectional type was used. A survey was applied and the Student $T$ was calculated from a database generated by the researcher from personal data records, academic performance and occupational categories to determine the effectiveness of the MIF according to the evaluation model of Donald Kirkpatrick and the quality criteria of ISO 9126. As results, it is evident that there is effectiveness in carrying out the MIF, it is evident that there was learning, as there was no statistically significant difference between the means obtained by the study subjects before and after Having completed the MIF, there are changes in behavior in the application of knowledge, skills and attitudes such as the practice of institutional values and a culture of service, as well as experience in conducting the course in a virtual environment where software complies with the requirements of ISO 9126. Therefore, use this model, as well as the criteria established that establishes ISO 9126, therefore, using both the Kirkpatrick model and ISO 9126 are a useful tool for organizations to determine the effectiveness of the training their collaborators receive. 


\section{Introducción}

En el año 2016 se creó la Gerencia de Formación de Personal SAT, con el propósito de capacitar y brindar experiencias formativas a todo el personal de la Superintendencia de Administración Tributaria, en diversas modalidades (Jiménez \& Barchino, 2014), para elevar las capacidades laborales de los colaboradores (Directorio de la Superintendencia de Administración Tributaria, 2016). El Módulo Introductorio de Formación (MIF) es uno de ellos, el cual se imparte en modalidad virtual a través de la plataforma educativa institucional (Moodle). El MIF lleva tres años de funcionamiento $y$, a pesar de ser un programa recurrente y automatizado, a la fecha no se ha evaluado cuál ha sido su efectividad, para conocer los alcances y el impacto que ha tenido en las personas que lo realizan.

Para determinar su efectividad se utilizó el modelo de evaluación de (Kirkpatrick \& Kirkpatrick, 2007) el cual sirve para evaluar acciones formativas basado en 4 niveles, siendo estos: reacción para saber la respuesta que tiene una persona al participar en un evento formativo, aprendizaje para evaluar los conocimientos, habilidades y actitudes adquiridas, conducta para evaluar si hubo un cambio en el comportamiento y resultado que es en conjunto, el resultado final de que la persona asista a un curso de formación.

Adicional a este modelo, se utilizó los criterios que establece la Norma ISO 9126, misma que sirve para evaluar un software desde su calidad interna y externa, a través de un conjunto de características que debe poseer un dicho software. (Alberto Ruiz, Gustavo, \& Peña, Alejandro, \& Arturo Castro, Carlos, \& Alaguna, Angela, \& Areiza, Luz Mery, \& Rincón, Rafael David, 2006) establecen que la calidad se determina en función de las características que hacen competitivo un producto al satisfacer las necesidades implícitas y explícitas de los usuarios finales. Derivado a que el Módulo Introductorio de Formación se desarrolla en una plataforma educativa Moodle, la evaluación de estos criterios se adapta a utilizar dicha norma.

Como parte del análisis de los resultados, se establece que el modelo de Kirkpatrick puede ser utilizado para evaluar 
programas completamente virtuales. Tal como lo indica (Jiménez, M. L., \& Barchino, R. P., 2014) este modelo es perfectamente trasladable a un entorno de aprendizaje virtual o e-learning, debido fundamentalmente a su sencillez y a su inmediata aplicabilidad. También se identifica la importancia de valorar el desempeño del software donde se desarrollan los programas formativos y el impacto que tiene en la experiencia del usuario cuando este cumple con los criterios que establece la ISO 9126.

\section{Materiales y métodos}

Se utilizó un enfoque cuantitativo, con alcance descriptivo, diseño no experimental transeccional. Las variables fueron: a) Nivel de efectividad, que tiene como dimensiones el modelo de evaluación de Donald Kirkpatrick y la norma ISO 9126, y

b) Categorías ocupacionales, que tiene como dimensiones Directivos e Intendentes, Jefes, Profesionales y Técnicos. Por otro lado, se planteó una hipótesis para comparar el desempeño de los participantes que aprueban el MIF antes y después de ser contratados en la SAT. Para poder comprobar dicha hipótesis se tiene como variable dependiente el rendimiento académico de la persona, que consiste en el promedio de notas obtenidas en el Módulo Introductorio de Formación y en el Programa de Inducción Institucional SAT. Mientras que la variable independiente se encuentra en la subcategoría ocupacional a la que pertenece el colaborador de SAT.

La población del estudio estuvo constituida por 715 colaboradores de SAT que realizaron el MIF. Se efectuó un muestreo probabilístico estratificado; los estratos fueron las dimensiones de la variable categorías ocupacionales. Para el cálculo de la muestra se utilizó un $95 \%$ de nivel de confianza y un $5 \%$ de error de estimación, lo que generó una muestra mínima de 251. Las unidades de análisis fueron elegidas aleatoriamente de un marco muestral constituido por todos los colaboradores que realizaron el MIF y que fueron contratados en la institución y para ello se realizó una proporción para cada estrato.

Se utilizaron los cuatro niveles que establece el modelo de Kirkpatrick para determinar el nivel de efectividad del MIF. Adicional al modelo, se usaron los criterios de la norma ISO 9126 para determinar el estándar de calidad del software donde se 
desarrolla el MIF. De acuerdo con (Abud Figueroa, 2004), esta norma establece que cualquier componente de la calidad del software puede ser descrito en términos de una o más de seis características básicas, las cuales son: funcionalidad, confiabilidad, usabilidad, eficiencia, mantenibilidad y portabilidad.

Se realizó el cálculo de la t de Studenten cada categoría ocupacional para comparar el rendimiento académico de los participantes con la base de datos de las notas obtenidas y visualizar si se acepta o rechaza la hipótesis nula.

\section{Resultados}

La encuesta permitió conocer el cumplimiento de los niveles del modelo de Donald Kirkpatrick, en tres de sus niveles (Reacción, Conducta y Resultado), así como la aplicación de los criterios de calidad de la ISO 9126. El nivel de aprendizaje del modelo de Donald Kirkpatrick fue evaluado en función de la base de datos de las notas obtenidas.

\section{Modelo de evaluación de Donald Kirkpatrick}

A continuación, se presentan los resultados de la evaluación que realizaron los encuestados del modelo de evaluación de Donald Kirkpatrick, en tres de sus niveles. Este resultado engloba las respuestas de todas las categorías ocupacionales.

Figura 1. Modelo de evaluación de Donald Kirkpatrick Nivel I, III y IV

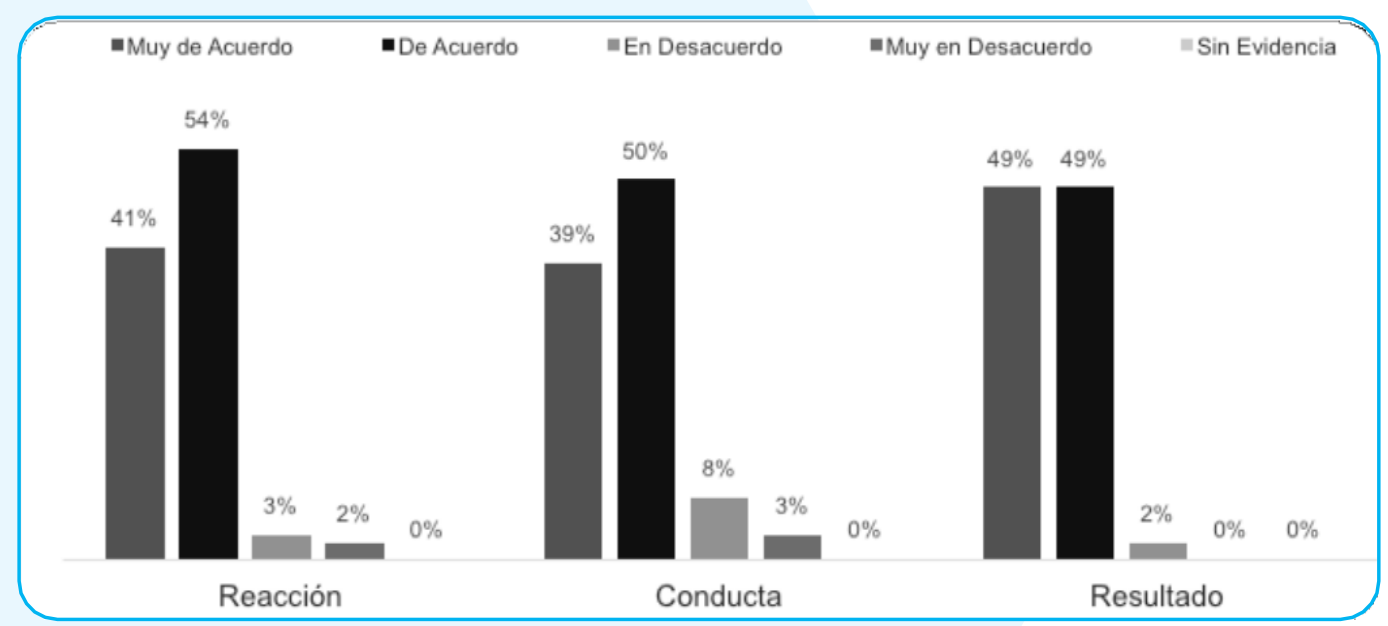

Fuente: Velásquez (2020). Datos obtenidos de la encuesta en línea realizada en diciembre 2019 a participantes que aprobaron el MIF y fueron contratados en la Institución a partir de enero del año 2018 a noviembre del año 2019. 
Para evaluar el Nivel 2 Aprendizaje se realizó el cálculo de la t de Student para determinar si hay una diferencia significativa entre las medias de las notas que obtuvieron los colaboradores que realizaron el Módulo Introductorio de Formación en las evaluaciones de los micromódulos de Conociendo la SAT, Ética y Valores y Atención al Contribuyente, así como en el Programa de Inducción Institucional SAT en esas mismas evaluaciones. De los datos calculados por subcategoría ocupacional se obtuvieron los siguientes resultados:

Tabla 1. Diferencia de medias en las categorías ocupacionales

\begin{tabular}{|c|c|c|c|c|}
\hline $\begin{array}{l}\text { Categoría } \\
\text { ocupacional }\end{array}$ & Subcategoría & $\begin{array}{l}\text { Sujetos de } \\
\text { estudio }\end{array}$ & t-Student & Prueba de hipótesis \\
\hline $\begin{array}{l}\text { Directivos e } \\
\text { Intendentes }\end{array}$ & Subgerente & 1 & $\mathrm{~N} / \mathrm{A}$ & No aplica \\
\hline \multirow[t]{2}{*}{ Jefe } & Jefe & 2 & 0.10 & Se acepta la hipótesis nula \\
\hline & Administrador & 2 & 0.40 & Se acepta la hipótesis nula \\
\hline \multirow[t]{2}{*}{ Profesional } & Supervisor & 5 & 0.44 & Se acepta la hipótesis nula \\
\hline & Profesional & 56 & 0.11 & Se acepta la hipótesis nula \\
\hline \multirow[t]{5}{*}{ Técnico } & Profesional & 64 & 0.39 & Se acepta la hipótesis nula \\
\hline & Especializado & 44 & 0.22 & Se acepta la hipótesis nula \\
\hline & Genérico & 64 & 0.06 & Se acepta la hipótesis nula \\
\hline & $\begin{array}{l}\text { Operativo } \\
\text { calificado }\end{array}$ & 8 & 0.21 & Se acepta la hipótesis nula \\
\hline & $\begin{array}{l}\text { Operativo no } \\
\text { calificado }\end{array}$ & 5 & 0.02 & Se rechaza la hipótesis nula \\
\hline
\end{tabular}

Fuente: Velásquez (2020). Con datos obtenidos de la base de datos de los participantes que aprobaron el MIF y fueron contratados en la Institución a partir de enero del año 2018 a noviembre del año 2019.

Cumplimiento de criterios ISO 9126

Resultados de la evaluación que realizaron los encuestados a la ISO 9126 en general de todas las categorías ocupacionales. 
Figura 2. Cumplimiento de Criterios ISO 9126

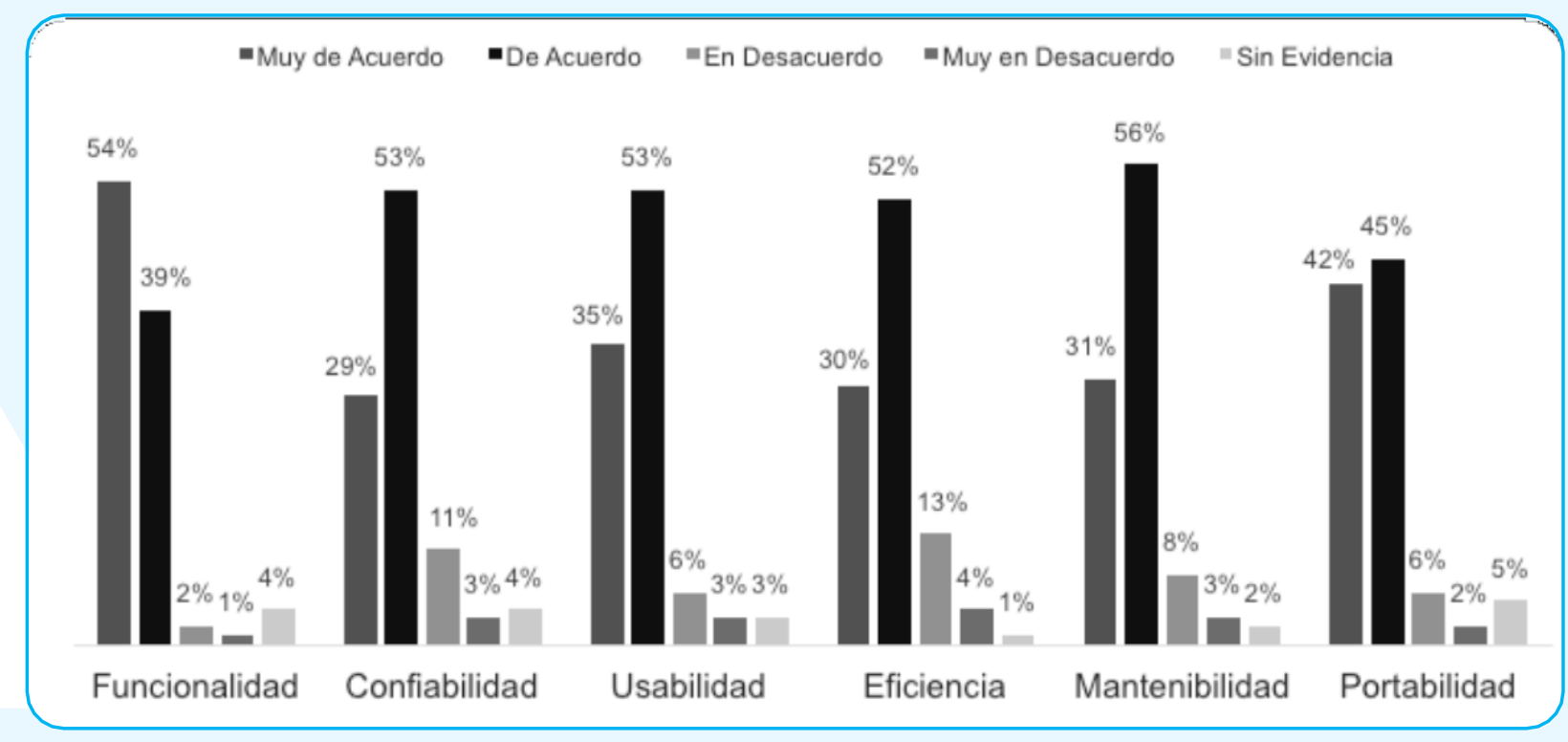

Fuente: Velásquez (2020). Datos obtenidos de la encuesta en línea realizada en diciembre 2019 a participantes que aprobaron el MIF y fueron contratados en la Institución a partir de enero del año 2018 a noviembre del año 2019

\section{Discusión}

En cuanto al modelo de evaluación de Donald Kirkpatrick, en este se refleja que cada nivel obtuvo valoración aceptable en las categorías ocupacionales de SAT; esto demuestra que se lograron los propósitos de formación planteados en el programa. Por otro lado, respecto al cumplimiento de los criterios de evaluación que indica la norma ISO 9126 de la plataforma educativa donde se desarrolla el MIF, se establece que la misma obtuvo aprobación en todos los criterios por parte de los participantes y que incide en la experiencia formativa al desarrollarse en un entorno virtual.

Con referencia de los resultados de la figura 1 y lo que establece el modelo de Kirkpatrick, para el nivel uno - Reacción, indica que, si la formación fue eficaz, es razonable que los estudiantes reaccionen favorablemente. Según (Kirkpatrick \& Kirkpatrick, 2007) "es importante obtener no solo una reacción, sino una reacción positiva"; es decir, que lo que se espera al evaluar una acción formativa es que todos o la mayoría de colaboradores hayan tenido una respuesta favorable al haber participado en un determinado curso. Mientras más positiva sea la reacción, 
mayor es el nivel de satisfacción que sienten al haberlo culminado. Esto demuestra que incide la presentación de los recursos en la plataforma educativa para que el participante tenga apertura y una reacción positiva durante el desarrollo de un curso.

En función de los datos de la Tabla 1, para evaluar el nivel dos - Aprendizaje de Kirkpatrick, se puede observar que al realizar el cálculo de la t de Student en las subcategorías ocupacionales, únicamente en la subcategoría de Operativo no Calificado se obtuvo un índice de 0.02 , lo que significa que la hipótesis nula se rechaza, es decir, que sí hay diferencia significativa entre el desempeño académico del colaborador cuando era candidato a una plaza en la SAT y realizó el MIF, comparado con el rendimiento académico que obtuvo posterior a su contratación. Mientras que, en las demás subcategorías, los índices que se obtuvieron están dentro de los rangos permitidos para aceptar la hipótesis nula y puede decirse que no hay diferencia estadísticamente significativa entre los resultados que obtuvo el colaborador antes y después de iniciar labores en SAT.

Respecto al nivel dos - Aprendizaje (Kirkpatrick \& Kirkpatrick, 2007) menciona que realizar esta evaluación permite medir la efectividad de un evento formativo para determinar si hubo un aumento en los conocimientos y/o cambios en las actitudes en las personas. Tomando como referencia los resultados de la Tabla 1 , puede decirse que los colaboradores de la SAT han adquirido aprendizajes cuando realizaron el MIF, los cuales fueron vueltos a evaluar cuando ingresaron a la institución y no hubo diferencia estadísticamente significativa entre su rendimiento académico.

Con relación al nivel tres - Conducta, (Kirkpatrick \& Kirkpatrick, 2007) establece que para conseguir que el cambio ocurra, son necesarias cuatro condiciones: que la persona tenga el deseo de cambiar, sepa lo que tiene que hacer y cómo hacerlo, trabaje en el clima adecuado y sea recompensada por el cambio. Por lo que, de acuerdo con los resultados de la figura 1, institucionalmente se cumple con las condiciones necesarias para que se produzca el cambio de conducta en los colaboradores, al contar con la voluntad y conocimiento por parte de la persona, así como el ambiente laboral y compensaciones que la institución ofrece. 
Dentro de los cambios de conducta que se fomentan en el MIF está la práctica de los valores institucionales que según la (Superintendencia de Administración Tributaria, 2008) en su Código de Ética y Conducta para el Personal de la SAT, los valores son la actitud de servicio, compromiso, productividad, respeto, responsabilidad y transparencia, así como mantener una cultura de servicio al ser una institución que atiende a contribuyentes.

En cuanto al nivel cuatro - Resultados, hubo una respuesta alta de aceptación de la efectividad del MIF, por lo que a nivel general cada nivel presenta una respuesta positiva y el objetivo ha sido logrado. Por último, (Guerra-López, 2007) menciona que, al utilizar este modelo, si los recursos humanos, financieros y temporales los permitan. Es importante evaluar cada nivel en orden para que todo tenga una secuencia lógica. De este modo se pueden identificar los puntos de mejora para la acción formativa de acuerdo a cada nivel e implementarlos en los siguientes cursos.

Respecto al cumplimiento de los criterios de la ISO 9126, cabe resaltar que el criterio con mejor evaluación fue el de funcionalidad, según la figura 2, que inicia desde que el participante ingresa al campus virtual y visualiza la página que le muestra el curso. De acuerdo con (Durán Portillo, 2015), la funcionalidad es cuando el software cumple eficazmente con las tareas para las que ha sido programado e ideado y para satisfacer las necesidades de sus usuarios. El criterio de funcionalidad en la plataforma educativa cumple con los atributos para su funcionamiento y para lo que fue diseñada; es decir, que sus funciones satisfacen las necesidades de sus usuarios. Este criterio es importante, por las expectativas que genera en los participantes, pues el presentar alguna dificultad en el ingreso, puede provocar un impacto negativo en la experiencia formativa.

También es importante reconocer qué tan confiable resulta el software que se utiliza y determinar los puntos críticos y de mejora. Para (Calderón Macías, 2016), la confiabilidad es el conjunto de atributos que tienen relación con la capacidad del software para mantener su nivel de funcionamiento durante un período establecido. Es decir, al hablar de confiabilidad se toma en cuenta la madurez para evitar fallos, ser tolerante 
ante algún error sin interrumpir su funcionamiento y restablecer su funcionamiento de forma rápida para evitar la pérdida de datos. De acuerdo con los resultados de la figura 2, sí se cumple con este criterio. Sin embargo, a pesar de haber sido bien evaluado, es importante tomar en cuenta acciones de mejora para aumentar la evaluación por parte de los usuarios.

Respecto al criterio de usabilidad, este se enfoca en el uso que los usuarios le dan al software, el cual depende del rol que tengan asignados. Según (Calderón Macías, 2016) la usabilidad es el conjunto de propiedades de un software que facilita al usuario trabajar con suficiente destreza e intuición. Este criterio evalúa la perspectiva desde los distintos roles del software para determinar el aprendizaje, la comprensión, la facilidad del uso e interfaz que presenta para los usuarios, pues ellos son los más indicados para valorar el criterio y si este cumple con sus expectativas. Según la figura 2, la plataforma cumple con las características de este criterio en cuanto al entendimiento que genera en el usuario al ser intuitiva y fácil de utilizar, lo que facilita el aprendizaje, resulta atractivo y amigable para el usuario.

En cuanto al criterio de eficiencia (Abud Figueroa, 2004) establece que esta característica permite evaluar la relación entre el nivel de funcionamiento del software y la cantidad de recursos usados. Este criterio establece dos características importantes: la primera es acerca del tiempo que demora el software para mostrar o reproducir material, ya sean imágenes, audios, videos, entre otros. La segunda es acerca de la cantidad de recursos o programas externos que el usuario necesita para poder visualizar el contenido del curso. De modo que, a nivel general, este criterio fue bien evaluado según la figura 2. En la encuesta se preguntó a los usuarios sobre la rapidez del software al navegar en la plataforma educativa y si los recursos se reprodujeron de forma adecuada; en todas las categorías ocupacionales hubo una aceptación del 82\%.

Para el criterio de mantenibilidad, (Durán Portillo, 2015) indica que esta puede encuadrarse en la vida que se le puede dar al producto o software, una vez creado; es decir, la capacidad que tiene dicho producto de ser actualizado o editado para mejorar, actualizar sus aplicaciones, cambiar una parte de la interfaz, etc. Un sistema o software es posible mantenerlo cuando responde 
ante una falla, modificación o actualización de forma rápida, por lo que en ese sentido, tanto jefes como profesionales y técnicos consideran que la plataforma educativa de la Gerencia, sí cumple con la mantenibilidad que establece la ISO 9126.

El criterio de portabilidad, de acuerdo con (Durán Portillo, 2015), indica que esta característica quizá sea la que más puede tener en cuenta los nuevos soportes y dispositivos que existen en la actualidad; es la capacidad que tiene el producto de ser adaptado a otros soportes. Con base en esto, los resultados de la figura 2 muestran que este criterio es adecuado para los usuarios, debido a que accedieron al campus virtual desde cualquier dispositivo con internet desde diversos sistemas operativos (Windows, Mac OC, Linux, Android, iOS, otro). Es importante resaltar que la plataforma educativa es de fácil acceso para que los usuarios ingresen desde cualquier sitio.

En conclusión, respecto al cumplimiento de los criterios de evaluación que indica la ISO 9126 de la plataforma educativa, se estableció que la misma obtuvo aprobación en todos los criterios evaluados. Por tanto, evaluar cuán efectivo es un programa de formación resulta de vital importancia para identificar el impacto que este provoca en las personas y cómo una institución se beneficia de ello. La aplicación de estos criterios en la plataforma educativa permite que este software se convierta en una herramienta útil para formar a personas de modo masivo y de fácil acceso.

\section{Agradecimientos}

Quiero extender un agradecimiento a la Gerencia de Formación de Personal SAT de la Superintendencia de Administración Tributaria, especialmente al Departamento de Formación Virtual por abrirme las puertas para realizar esta investigación y brindar toda la información para llegar a estas conclusiones. Agradezco al maestro Edwing Roberto García, Dra. Miriam Judith Hernández Rivera y Dra. Patricia Luz Mazariegos Romero por su apoyo incondicional durante el desarrollo de este estudio en sus distintas etapas. 


\section{Referencias}

Abud Figueroa, M. A. (2004). Calidad en la industria del software. La norma ISO-9126. Revista UPIICSA, 3. Recuperado el 29 de febrero de 2020. Disponible en: http://148.204.210.204/revistaupiicsa/34/34-2.pdf

Alberto Ruiz, G., \& Peña, A., \& Arturo Castro, C., \& Alaguna, A., \& Areiza, L.M., \& Rincón, R.D. (2006). Modelo de Evaluación de Calidad de Software Basado en Lógica Difusa, Aplicada a Métricas de Usabilidad de Acuerdo con la Norma ISO/IEC 9126. Revista Avances en Sistemas e Informática, 3(2),25-29. Recuperado el 28 de mayo de 2020, ISSN: 1657-7663. Disponible en: https://www.redalyc.org/articulo. oa? id=1331/133114988005

Calderón Macías, F. R. (2016). El estándar ISO y su aportación al proceso de calidad del desarrollo de software. Recuperado el 28 de febrero de 2020. Disponible en: http://openaccess.uoc.edu/webapps/o2/ bitstream/10609/53422/8/fcalderonmTFC0616memoria.pdf

Directorio de la Superintendencia de Administración Tributaria. (2016). Acuerdo de Directorio Número 014-2016 Reformas al Acuerdo de Directorio Número 007-2007, Reglamento Interno de la Superintendencia de Administración Tributaria.

Directorio de la Superintendencia de Administración Tributaria. (2018). Acuerdo de Directorio Número 033-2018, Código de Ética y conducta para el Personal de la SAT.

Durán Portillo, D. (2015). Gestión de la calidad de productos editoriales multimedia. ARGN0110 (1ra. edición ed.). IC Editorial. Recuperado el 12 de febrero de 2020. Disponible en: https://books.google.com. $\mathrm{gt} / \mathrm{books}$ ? id=q2k7DwAAQBAJ\&printsec=frontcover\&hl=es\#v=onepage \&q\&f=false

Guerra-López, I. (2007). Evaluación y mejora continua: conceptos y herramientas para la medición y mejora del desempeño. AuthorHouse. Recuperado el 14 de marzo de 2020. Disponible en: https://books.google.com.gt/ books?id=tQiAlcui5dsC \& printsec=frontcover $\&$ hl=es\#v=onepage $\& q \& f=-$ false

Jiménez, M. L., \& Barchino, R. P. (15 de julio de 2014). Evaluación e implantación de un modelo de evaluación de acciones formativas. Recuperado el 01 de marzo de 2020. Disponible en: https://www.researchgate.net/ publication/228854838_Evaluacion_e_implantacion_de_un_modelo_de_ evaluacion_de_acciones_formativas

Kirkpatrick, D. L., \& Kirkpatrick, J. D. (2007). Evaluación de acciones formativas: los cuatro niveles (3ª edición). Grupo Planeta (GBS). 


\section{Sobre la autora}

Lidia Velásquez es Licenciada en Pedagogía y Administración Educativa. Cierre de pensum de Maestría en Investigación en la Universidad de San Carlos de Guatemala. Diseñadora de Formación Virtual en la Superintendencia de Administración Tributaria.

Copyright (c) Lidia Velásquez, Miriam Hernández y Patricia Mazariegos

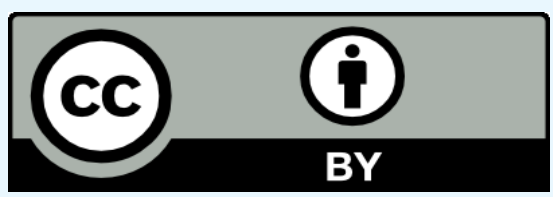

Este texto está protegido por una licencia CreativeCommons 4.0.

Usted es libre para compartir, copiar y redistribuir el material en cualquier medio o formato y adaptar el documento, remezclar, transformar y crear a partir del material para cualquier propósito, incluso comercialmente, siempre que cumpla la condición de atribución: usted debe reconocer el crédito de una obra de manera adecuada, proporcionar un enlace a la licencia, e indicar si se han realizado cambios. Puede hacerlo en cualquier forma razonable, pero no de forma tal que sugiera que tiene el apoyo del licenciante o lo recibe por el uso que hace. 